\title{
Study of adaptive Fuzzy Smith control for time-delay systems
}

- Nguyen Trong Tai

- Dao Van Thanh

Ho Chi Minh city University of Technology, VNU-HCM, Vietnam

(Manuscript Received on July 15, 2015, Manuscript Revised August 30, 2015)

\section{ABSTRACT}

In this paper, an adaptive Fuzzy Smith control method is presented to control the varying time delay systems. Based on the online parameter estimation, Smith predictor can be updated online which can eliminate the time delay element. This method overcame the shortcomings that control effect of conventional Smith predictor will be worse when the parameters of time delay systems change. Furthermore, an adaptive fuzzy controller adjusts online the PID control parameters to improve the control performance. Simulation results show the effectiveness of the proposed method.

Keywords: Adaptive control, fuzzy control, Smith predictor, time delay system.

\section{INTRODUCTION}

In industrial processes, time delay is a common phenomenon in the controlled object. Because of this phenomenon, the dynamic error will increase, which having a significant effect on performance of the control systems. Many control algorithms such as PID with fixed parameters are often unsuitable for such processes, especially when time delay and other parameters of the system are unknown and changed.

Smith predictor is an effective method to control a process when parameters and time delay are known and fixed. However, Smith predictor is more sensitive to process parameter variations. If the process model mismatch, the control performence of the system will be deteriorated even leads the system to instability. Significant researchs relate to the robustness issues of the Smith predictor system. Just to mention a few, to improve the robust performance of Smith predictor within the IMC [1]. The tuning of Smith predictor when plant time delay is not precisely known [2]. A Toolbox in the MATLAB environment was designed for identification and self-tuning control of such processes [3].

This paper proposes an approach that can extend the Smith predictor to a wide range of the time-varying processes with dominant and variable time delay. The time delay parameter in the predictor model is estimated online by minimizing the error between model output and process model [4], and other variable parameters of the predictor model is also tuned by online parameter estimation.

To improve the control performance further, an adaptive fuzzy controller is also introduced and discussed in $[5,6]$. The proposed approach automatically adjusts the PID parameters to adapt to the time-varying systems. The results have 
demonstrated that the proposed approach is effective.

\section{STRUCTURE OF CONTROL SYSTEM}

Consider the process with time-varying delay as described by the ARMAX (regression) model:

$$
A(q) y(k)=q^{-d(k)} B(q) u(k)+C(q) e(k)(1)
$$

Where:

$$
\begin{aligned}
& A(q)=1+a_{1} q^{-1}+\ldots+a_{n a} q^{-n a} \\
& B(q)=b_{0}+b_{1} q^{-1}+\ldots+b_{n b} q^{-n b} \\
& C(q)=1+c_{1} q^{-1}+\ldots+c_{n c} q^{-n c}
\end{aligned}
$$

With $a_{1}, \ldots, a_{n a}, b_{0}, \ldots, b_{n b}, c_{1}, \ldots, c_{n c}$ are the timevarying parameters of the system, $d_{\min } \leq d(k) \leq$ $d_{\text {max }}$ is the time-varying delay, $u(k), y(k)$, and $e(k)$ are controller output, system output and white noise serial, respectively.

By minimizing the error between the process output and the process model output and online parameter estimation, Smith predictor will be updated online. Furthermore, to improve the control requirement, an adaptive fuzzy controller which can online adjust the scaling factors of the PID controller is also addressed. The structure of the control system is introduced in Fig 1.

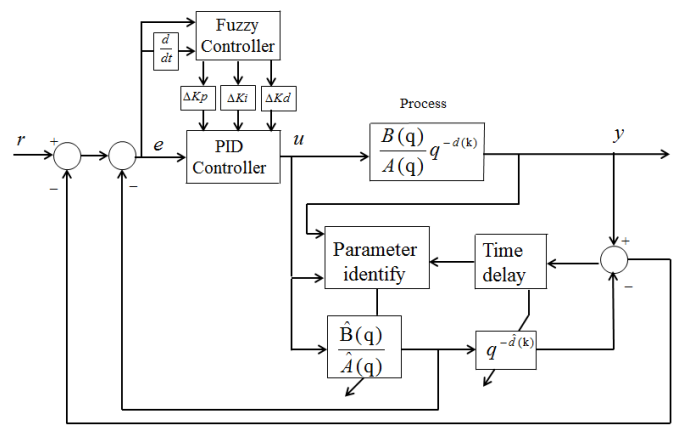

Fig 1: Block diagram of control system.

\section{ADAPTIVE SMITH PREDICTOR}

Assume that the process model is given by:

$$
\hat{A}(q) y(k)=q^{-\hat{d}(k)} \hat{B}(q) u(k)+\hat{C}(q) e(k)
$$

Where:

$$
\begin{aligned}
& \hat{A}(q)=1+\hat{a}_{1} q^{-1}+\ldots+\hat{a}_{n a} q^{-n a} \\
& \hat{B}(q)=\hat{b}_{0}+\hat{b}_{1} q^{-1}+\ldots+\hat{b}_{n b} q^{-n b} \\
& \hat{C}(q)=1+\hat{c}_{1} q^{-1}+\ldots+\hat{c}_{n c} q^{-n c}
\end{aligned}
$$

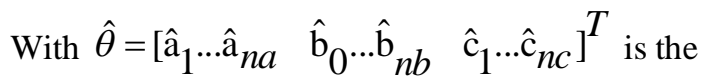
estimation of the parameters

$\theta=\left[\begin{array}{lll}\mathrm{a}_{1} \ldots \mathrm{a}_{n a} & \mathrm{~b}_{0} \ldots \mathrm{b}_{n b} & \mathrm{c}_{1} \ldots \mathrm{c}_{n c}\end{array}\right]^{T}$ at time $k, \hat{d}(\mathrm{k})$ is the estimation of the time delay $d(k)$.

\subsection{Identification algorithm of the time} delay

The error between the process output and the estimation output can be defined as:

$$
\begin{aligned}
& \varepsilon(k)=y(k)-\hat{y}(k, \theta) \\
& =u(k)\left[\frac{B(q)}{A(q)} q^{-d(k)}-\frac{\hat{B}(q)}{\hat{A}(q)} q^{-\hat{d}(k)}\right] \\
& +e(k)\left[\frac{C(q)}{A(q)}-\frac{\hat{C}(q)}{\hat{A}(q)}\right]
\end{aligned}
$$

The cost function for parameter identification is defined as:

$$
J(\mathrm{k})=\sum_{i=1}^{k} \frac{1}{2} \varepsilon^{2}(\mathrm{i})
$$

Assume $\hat{\theta}=\theta, \quad \hat{d}(\mathrm{k}) \neq \mathrm{d}(\mathrm{k})$ to minimise the cost function $J(k)$, the time delay $\hat{d}(\mathrm{k})$ can be chosen as:

$$
\hat{d}(\mathrm{k})=\hat{\mathrm{d}}(\mathrm{k}-1)-\left.\mathrm{m}(\mathrm{k}) \frac{\partial J}{\partial \hat{d}(\mathrm{k})}\right|_{\hat{d}=\hat{d}(\mathrm{k})}
$$

Where $m(k)$ is the optimum step size. 


$$
\begin{aligned}
& \frac{\partial J}{\partial \hat{d}(\mathrm{k})}=\sum_{i=1}^{k} \varepsilon(\mathrm{i}) \mathrm{u}(\mathrm{i}) \frac{\hat{B}(\mathrm{q})}{\hat{A}(\mathrm{q})} q^{-\hat{d}(\mathrm{i})} \ln q \\
& =\left.\sum_{i=1}^{k} \varepsilon(\mathrm{i}) \hat{\mathrm{y}}(\mathrm{i}) \mathrm{sT}\right|_{S=\frac{1-q^{-1}}{T}} \\
& =\sum_{i=1}^{k} \varepsilon(\mathrm{i})[\hat{y}(\mathrm{i})-\hat{\mathrm{y}}(\mathrm{i}-1)]
\end{aligned}
$$

Therefore, the identification algorithm of the time delay is:

$$
\begin{aligned}
& \hat{d}(\mathrm{k})=\hat{\mathrm{d}}(\mathrm{k}-1)-\Delta \hat{\mathrm{d}}(\mathrm{k}) \\
& =\hat{\mathrm{d}}(\mathrm{k}-1)-\mathrm{m}(\mathrm{k}) \sum_{i=1}^{k} \varepsilon(\mathrm{i})[\hat{y}(\mathrm{i})-\hat{\mathrm{y}}(\mathrm{i}-1)] \quad(5) \\
& \text { Where } m(\mathrm{k})=\frac{1-\alpha}{\sum_{i=1}^{k}[\hat{y}(\mathrm{i})-\hat{\mathrm{y}}(\mathrm{i}-1)]^{2}}, 0 \leq \alpha \leq 1
\end{aligned}
$$

Noticed that, $\Delta \hat{d}(\mathrm{k})$ should be converted to an integer so that $\hat{d}(\mathrm{k})$ can be an integer.

\subsection{Identification algorithm of time- varying parameters}

Parameter vector $\hat{\theta}$ can be estimated online by the least squares method after time delay is identified. The structure of the process model into the least squares method is:

$$
\hat{y}(k, \theta)=\varphi^{T}(k, \theta) \theta+e(k)
$$

Where

$$
\begin{aligned}
& \varphi(k, \theta)=\left[-y(k-1) \ldots-y\left(k-n_{a}\right) \quad u(k-\hat{d}(k)) \ldots\right. \\
& \left.u\left(k-\hat{d}(k)-n_{b}\right) \quad e(k-1) \ldots e(k-n c)\right]^{T}
\end{aligned}
$$

Many amelioration approachs based on the least square method is used to estimating parameter in [7]. In this paper, we adopt the recursive identification algorithm.

$$
\begin{aligned}
\hat{\theta}(\mathrm{k}) & =\hat{\theta}_{k-1}+L(\mathrm{k}) \varepsilon(\mathrm{k}) \\
\varepsilon(\mathrm{k}) & =\mathrm{y}(\mathrm{k})-\varphi^{T}(\mathrm{k}) \hat{\theta}(\mathrm{k}-1) \\
L(\mathrm{k}) & =\frac{P(\mathrm{k}-1) \varphi(\mathrm{k})}{\lambda(\mathrm{k})+\varphi^{T}(\mathrm{k}) P(\mathrm{k}-1) \varphi(\mathrm{k})} \\
P(\mathrm{k}) & =\frac{1}{\lambda(\mathrm{k})}\left[P(\mathrm{k}-1)-\frac{P(\mathrm{k}-1) \varphi(\mathrm{k}) \varphi^{T}(\mathrm{k}) \mathrm{P}(\mathrm{k}-1)}{\lambda(\mathrm{k})+\varphi^{T}(\mathrm{k}) P(\mathrm{k}-1) \varphi(\mathrm{k})}\right]
\end{aligned}
$$

Where

$\lambda$ is called the forgetting factor and typically has a positive value between $0.95 \div 0.998$.

Assume initial values are $P(0)=\sigma^{2} I$ where $\sigma^{2}$ is a sufficient numeral, $\hat{\theta}$ is a offline identification value and $I$ is an identity matrix.

\section{DESIGN OF CONTROLLER}

To overcome the shortcomings of Smith predictive control, the advantage that fuzzy control can achieve good control effects without the accurate math model of controlled plant is used. In this paper, the fuzzy control is combined with Smith predictive control and PID control to construct the fuzzy PID controller. Its block diagram is also shown in Fig1.

The fuzzy controller has two inputs that are the error $e$ and the deviation of the error $d e$ respectively, three outputs that are the regulating factors $\Delta K p, \Delta K i$ and $\Delta K d$. The tuning arithmetic of the parameters as follows:

$$
\begin{aligned}
& K p=K p^{\prime}+\Delta K p \\
& K i=K i^{\prime}+\Delta K i \\
& K d=K d^{\prime}+\Delta K d
\end{aligned}
$$

Where $K p^{\prime}, K i^{\prime}$, and $K d^{\prime}$ are the initial values of the PID controller.

According to the requirement of PID control and control experience, the fuzzy control rules of $\Delta K p, \Delta K i, \Delta K d$ are defined. Take $\Delta K p$ for example, the fuzzy control rule tables are shown in Table 1. The weighted average method is employed to de-fuzz the output variation. 


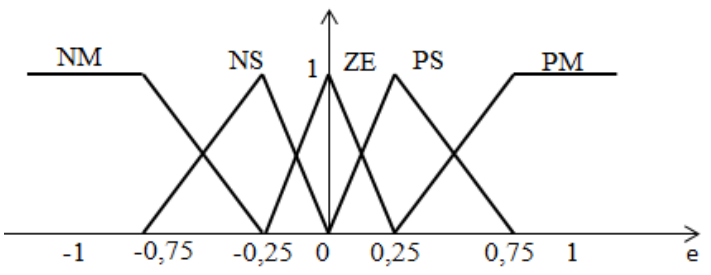

Fig 2: Membership functions of $e$

The language values of $e$, $d e$ are defined as follows: The characters NM, NS, ZE, PS, PM, $\mathrm{NE}$, and PE stand for negative medium, negative small, zero, positive small, positive medium, negative, and positive.

The membership functions of $e$ and $d e$ are shown in Fig 2 and Fig 3.

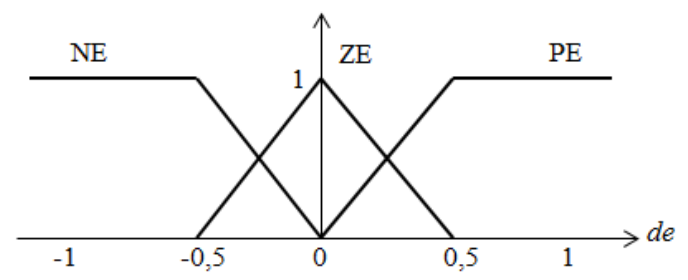

Fig 3: Membership functions of $d e$

The language values of $\Delta K p$ are defined as follows: The characters ZE, PE, PS, PM, and PB stand for zero, positive, positive small, positive medium and positive big.

The membership functions of $\triangle K p$ are shown in Fig 4.

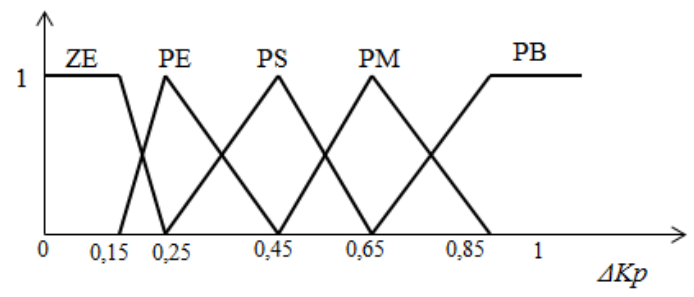

Fig 4: Membership functions of $\Delta K p$

The table of fuzzy rules appear as follows:

Table 1: Fuzzy rules of $\Delta K p$

\begin{tabular}{|l|l|l|l|l|}
\hline \multicolumn{2}{|c|}{$\Delta K p$} & \multicolumn{3}{c|}{$d e$} \\
\cline { 3 - 5 } & $\mathrm{NE}$ & $\mathrm{ZE}$ & $\mathrm{PE}$ \\
\hline$e$ & $\mathrm{NM}$ & $\mathrm{PB}$ & $\mathrm{PM}$ & $\mathrm{PS}$ \\
\hline
\end{tabular}

\begin{tabular}{|l|l|l|l|l|}
\hline NS & PS & PE & PE \\
\cline { 2 - 5 } & ZE & ZE & ZE & PE \\
\cline { 2 - 5 } & PS & PS & PS & PM \\
\cline { 2 - 5 } & PM & PS & PM & PB \\
\hline
\end{tabular}

\section{SIMULATION RESULTS}

The process that is chosen to simulate is the single tank as Fig 5. Where the time delay is changed due to the sensor and transmistion time.

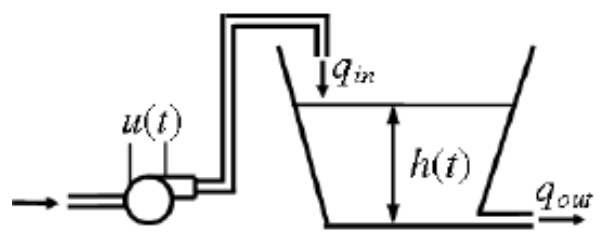

Fig 5: Single tank

The differential equation without the time delay that describes the dynamic characteristic of the process is :

$$
\dot{h}(\mathrm{t})=\frac{(\mathrm{ku}(\mathrm{t})-b \mathrm{C} \sqrt{2 g h(\mathrm{t})})}{\left(\mathrm{A}_{\max }-\mathrm{A}_{\min }\right) \mathrm{h} / \mathrm{h}_{\max }+A_{\min }}
$$

Where

$\mathrm{A}_{\max }$ : Maximum cross area $\left(\mathrm{cm}^{2}\right)$

$\mathrm{A}_{\min }$ : Minimum cross area $\left(\mathrm{cm}^{2}\right)$

b: Outlet area $\left(\mathrm{cm}^{2}\right) ; \mathrm{g}=981 \mathrm{~cm} / \mathrm{s}^{2}$

C: Discharge constant

$\mathrm{k}$ : Scaling factor with pump capacity

$\mathrm{u}(\mathrm{t})$ : Control voltage $(\mathrm{V})$

$\mathrm{h}(\mathrm{t})$ : liquid height

$\mathrm{h}_{\max }$ : Maximum tank height

The regression vectors of $\varphi$ and $\hat{\theta}$ are chosen as follows:

$$
\begin{aligned}
& \varphi=\left[\begin{array}{lll}
-y(\mathrm{k}-1) & -\sqrt{y(\mathrm{k}-1)} & u(\mathrm{k}-\hat{\mathrm{d}}-1)
\end{array}\right]^{T} \\
& \hat{\theta}=\left[\begin{array}{lll}
\hat{a}_{1} & \hat{a}_{2} & \hat{b}_{1}
\end{array}\right]^{T}
\end{aligned}
$$


In simulation block diagram is combined with the anti-windup as described in Fig 6.

In the first simulation, the system parameters are unchange and the time delay is set $\tau_{\mathrm{d}}=1 \mathrm{~s}$. After $600 \mathrm{~s}$, increasing time delay by $7 \mathrm{~s}\left(\tau_{\mathrm{d}}=8 \mathrm{~s}\right)$.

For comparative study, two methods are used in simulation. One is PID conventional, the other is fuzzy Smith predictor addressed in this paper.

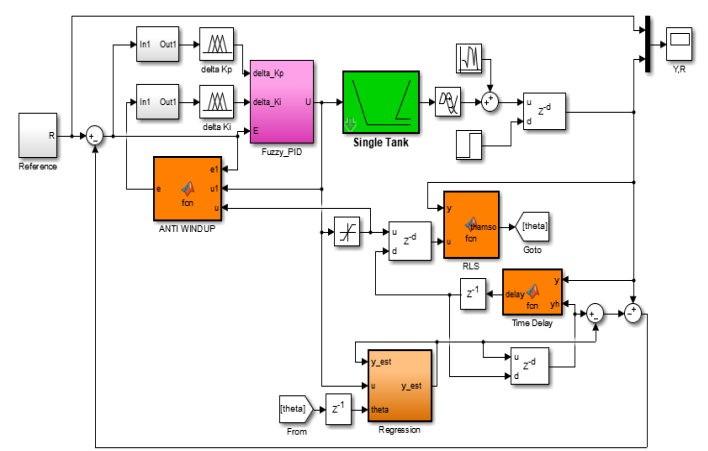

Fig 6: Simulation block diagram

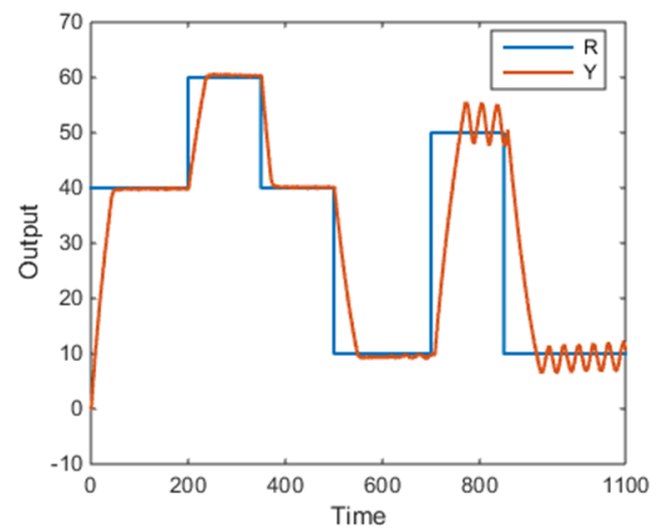

Fig 7: System respone with PID conventional

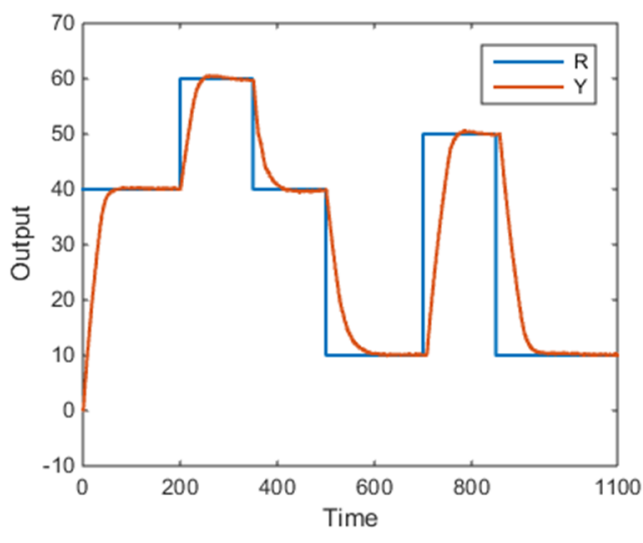

Fig 8: System respone with fuzzy Smith controller

The control results are shown in Fig 7 and Fig 8. The method one exhibits oscilation and instability. However, by using identification algorithms online and fuzzy logic control to adjust PID parameter online, the method two can eliminate the time delay element and cancel oscillation effectively and make the system to stabilization.

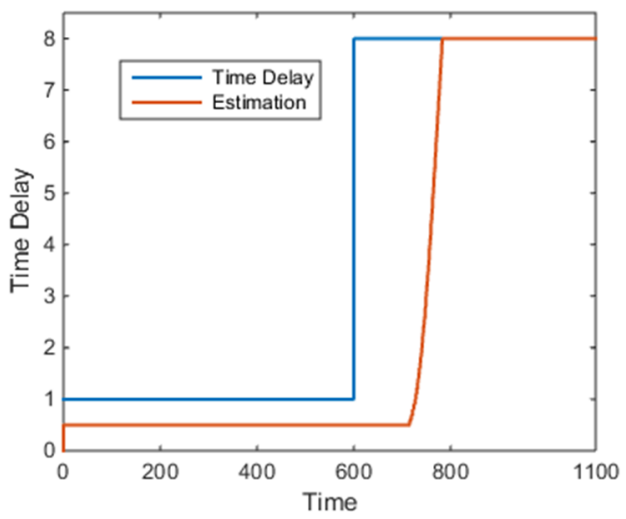

Fig 9: Estimaiton result of time delay

As shown in Fig 9, when the time delay changes from $\tau_{\mathrm{d}}=1 \mathrm{~s}$ to $\tau_{\mathrm{d}}=8 \mathrm{~s}$ at time $600 \mathrm{~s}$, the estimation time delay converges to the process delay and then tracks it, ensuring control result.

In the second simulation, the model parameters are changed as follows: Initially, the system time delay $\tau_{\mathrm{d}}=1 \mathrm{~s}$ and discharge constant 
$\mathrm{C}=0.6$. After $600 \mathrm{~s}$, increasing discharge constant $\mathrm{C}$ by $0.25(\mathrm{C}=0.85)$ and time delay by $7 \mathrm{~s}\left(\tau_{\mathrm{d}}=8 \mathrm{~s}\right)$.

For comparative study, two controllers are used in this simulation. The one is combination of Smith predictor and PID controller, the another is the fuzzy Smith controller proposed in this study.

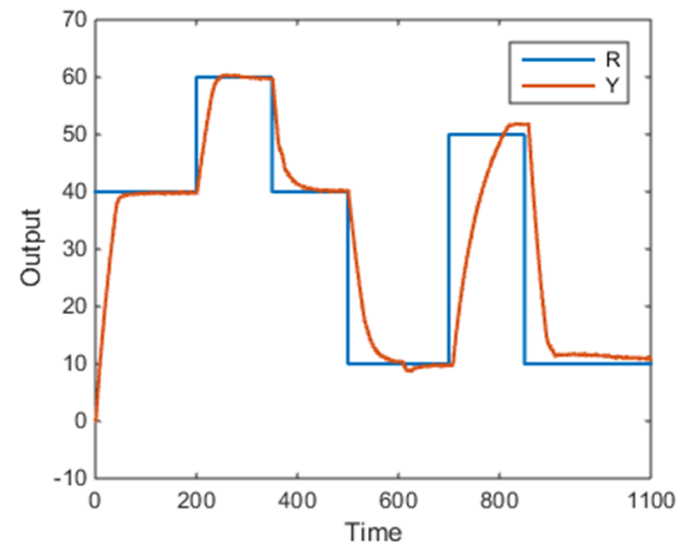

Fig 10: Respone with PID Smith controller

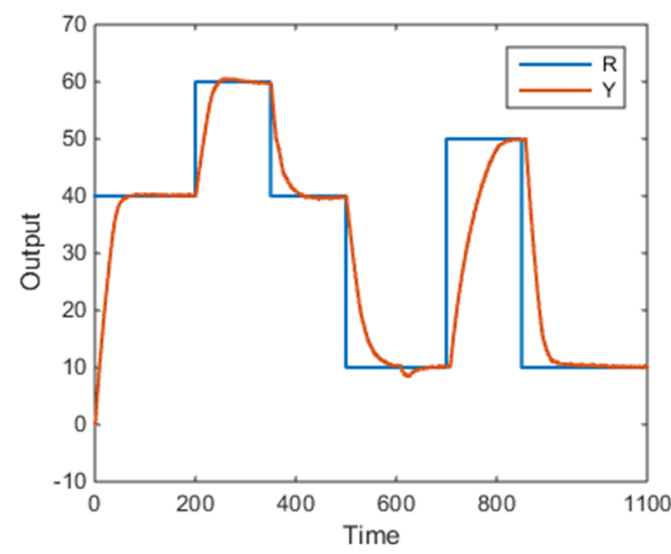

Fig 11: Respone with Fuzzy Smith controller

As shown in Fig 10 and Fig 11, fuzzy Smith controller is better than PID Smith controller in the performance index of steady-state error.

\section{CONCLUSION}

The identification algorithm is used to estimate online the parameters and the time delay of the system. Therefore, it can overcome the inherent drawback of the Smith predictor. The apdaptive fuzzy Smith method proposed in this paper can adjust the PID parameters online. Simulation results demonstrate the effectiveness of this method for various conditions.

\section{ACKNOWLEDGEMENT}

This research is funded by Vietnam National University HoChiMinh City (VNU-HCM) under grant number C2014-20-29. 


\section{Điều khiển thích nghi Smith mờ cho hệ thống có thời gian trễ}

- Nguyễn Trọng Tài

- Đào Văn Thành

Trường Đại học Bách Khoa, ĐHQG-HCM, Việt Nam

\section{TÓM TÁT}

Bộ điều khiển thích nghi Smith mờ được đề xuất trong bài báo này được dùng để điều khiển những hệ thống có thời gian trễ khi những tham số và thời gian trễ của hệ thống thay đổi. Dựa trên việc ước lượng những tham số online, bộ dụ̂ báo Smith được cập nhật online sẽ loại bỏ ảnh hưởng của thời gian trễ lên hệ thống. Phương pháp này có thể khắc phục những thiếu sót của bộ dụ̂ báo Smith offline khi tham số của đối tượng thay đổi. Ngoài ra, bộ điều khiển mờ thích nghi sẽ chỉnh online những tham số của bộ điều khiển PID để cải thiện chất lượng hệ thống. Kết quả mô phỏng cho thấy hiệu quả của phương pháp được đề xuất.

Từ khóa: Điều khiển thích nghi, điều khiển mờ, bộ dụ̣ báo Smith, hệ thống có trễ.

\section{REFERENCES}

[1]. D.L. Laughlin, D.E. Rivera, and M. Morari, "Smith predictor design for robust performance", Int. J. Control, vol. 46, no.2, pp. 477-504, 1987.

[2]. C. Santacesaria and R. Scattolini, "Easy tuning of Smith predictor in presence of delay uncertainty", Automatica, 29, pp. 1595-1597, 1993.

[3]. Vladimír Bobál, Petr Chalupa, Marek Kubalčík, Petr Dostál, "Identification and self-tuning control of time-delay systems", Wseas transactions on systems, Issue 10, Volume 11, October 2012.

[4]. Wei Gao, Yuanchun Li, Guangjun Liu, and Tao Zhang, "An adaptive fuzzy Smith control of time-varying processes with dominant and variable delay", Proceedings of the American control Conference Denver, Colorado June 4$6,2003$.

[5]. Wang Ke, Luo Wenguang, Shi Yuqiu, Li Xiaofeng, "Study of self-adaptive fuzzy Smith control for liquid level systems", 2010 International conference on measuring technology and mechatronics automation.

[6]. Liu Wen-bo, Wang Meng-xiao, "Research on fuzzy Smith control method for time delay systems", 978-1-4244-5874-5/10/2010 IEEE.

[7]. Lennart Ljung, "System identification: Theory for the User", Prentice Hall, pp. 303305, 1987. 